\title{
Mini review - Clinical and physiological aspects of pain in parkinson's disease
}

\author{
Eduardo NC Bergamaschi ${ }^{1}$, Fernanda C Nunes ${ }^{2,3}$, Victor W de Oliveira ${ }^{3}$, Alessandra Laitart ${ }^{3}$, Maria L Bene- \\ vides $^{3}$, Jean C Nunes ${ }^{2,3}$ \\ 'Serviço de Neurologia, Departamento de Clínica Médica, Universidade Federal de Santa Catarina (UFSC), Florianópolis, SC, Brazil. \\ ${ }^{2}$ Neurodiagnostic Brasil, Diagnósticos em Neuropatologia, Florianópolis, SC, Brazil. \\ ${ }^{3}$ Division of Neuropathology, Hospital Universitário (HU), UFSC, Florianópolis, SC, Brazil
}

\section{Article Info}

\section{Article Notes}

Received: July 25, 2016

Accepted: August 25, 2016

\section{*Correspondence: \\ Jean Costa Nunes, M.D., Ph.D., Division of Neuropathology, Serviço de Anatomia Patológica, 1st floor, Hospital Universitário Polydoro Ernani de São Thiago, Universidade Federal de Santa Catarina (UFSC), Rua Professora Maria Flora Pausewang, Trindade, Florianópolis, Santa Catarina (SC), Brazil, Zip Code: 88040-000; Telephone: +55 4891641334; Fax number: +55 4837218045;E-mail: jeanmedic@hotmail.com}

(c) 2016 Nunes JC. This article is distributed under the terms of the Creative Commons Attribution 4.0 International License

\section{Keywords}

Parkinson's disease

Headache

Pain

NMS

Deep brain stimulation

\section{ABSTRACT}

Pain is a common and often disabling symptom in Parkinson's disease (PD) which has received increasing attention in recent years. Headache represents a common form of pain among the general population, but there are few studies on this symptom in PD. In 2014, our group reported a lower prevalence of headache in PD patients compared to the general population, as well as an association between the predominant side of headache and the side of initial motor signs of PD. Since then, there has been few new data on the specific issue of headache in PD patients, though several recent studies have contributed to the understanding of pain in PD, both in terms of clinical and pathophysiological aspects, including new observations on pain association with side of motor symptom onset. Here, we review those studies, and re-discuss our own findings in comparison to the current information available. A better comprehension of pain physiology in PD could facilitate the development of new therapeutic approaches, thus providing a better quality of life for PD patients.

\section{Introduction}

Pain is a common and often disabling symptom in Parkinson's disease (PD) ${ }^{1}$. Headache represents a common form of pain, but there are few studies about headache in $\mathrm{PD}^{2}$. In an article published in 2014, we reported the prevalence and characteristics of headache in 100 consecutive PD patients 2 . They were compared to 98 ageand gender-matched controls from a Brazilian population-based epidemiological study ${ }^{2,3}$. We found a statistically significant lower prevalence of headache in PD patients. It was also shown that, when the PD patients' headache was unilateral, its predominant side was ipsilateral to the side of initial motor signs of PD, with $84 \%$ concordance $(p<0.01)^{2}$.

Although the study design did not allow a cause-effect relationship, we hypothesized that this association could be explained by some protective effect of PD against headache. Since then, many works have addressed the issue of pain in $\mathrm{PD}^{4-17}$. Here, we reviewed the new findings about pain in PD, and re-discuss our hypothesis in the light of the current information.

\section{Pain in Parkinson's disease}

Pain in PD is frequent $t^{1,4-6}$, can be disabling ${ }^{1}$ and have an important impact on the patients' quality of life ${ }^{4}$. Pain is reported among the symptoms of PD since the earliest publications on the subject, and 


\begin{tabular}{|c|c|c|c|c|c|}
\hline Reference & $\begin{array}{c}\text { Musculoskeletal } \\
\%\end{array}$ & $\begin{array}{c}\text { Radicular/neuropathic } \\
\%\end{array}$ & $\begin{array}{l}\text { Dystonic } \\
\%\end{array}$ & $\begin{array}{c}\text { Central/primary } \\
\%\end{array}$ & $\begin{array}{c}\text { Akathisia } \\
\%\end{array}$ \\
\hline Valkovic $\mathrm{P}$ et $\mathrm{al}^{4}$ & 41.0 & 27.0 & 17.0 & 22.0 & a \\
\hline Rana A et al ${ }^{5}$ & 28.0 & 36.0 & 48.0 & $b$ & 29.0 \\
\hline Mao CJ et $a^{6}$ & 47.1 & 19.1 & 23.5 & 7.4 & 2.9 \\
\hline
\end{tabular}

${ }^{\text {a Reference }}{ }^{4}$ did not assess akathisia

${ }^{\text {b }}$ Reference ${ }^{5}$ did not describe the prevalence for central/primary pain

Table 1: Relative frequency of each category of pain in Parkinson's disease (Ford's classification).

is noted for being capable of overshadowing the motor symptoms of the disease ${ }^{1}$. A recent study reported a significant association between pain and poorer quality of life and more severe depression in PD patients ${ }^{4}$. Therefore, pain emerges as an important non-motor symptom (NMS) of PD, albeit an often overlooked one ${ }^{1}$.

In order to provide better diagnosis and comprehension of pain in PD, several pain classification systems in PD have been designed ${ }^{4}$, of which the system proposed by Ford $^{1}$ remains the most cited ${ }^{4}$. The author classified pain in PD in five categories: musculoskeletal, radicular/neuropathic, dystonic, central/primary and akathisia ${ }^{1}$. According to this system, PD-related headache is classified as central/ primary pain ${ }^{1}$. This scheme was used in several papers reviewed here ${ }^{4-6}$, and the relative frequency of each category of pain is exposed in Table 1.

\section{Prevalence studies}

Although no new published research has specifically assessed the frequency and characteristics of headache in PD, many studies in recent years have investigated the prevalence of pain among PD patients and factors that could be related to its occurrence ${ }^{4-6}$. Valkovic et al. reported a $76.0 \%$ prevalence of overall pain (OP) among PD patients in a cross-sectional study, though headache was not referred in this population ${ }^{4}$. OP was found to be worse in patients with advanced PD, with patients with advanced disease reporting more frequently musculoskeletal, radicular/ neuropathic, dystonic and central/primary pain ${ }^{4}$. Patients with early PD reported more frequently pain that could not be classified under those categories, thus being put

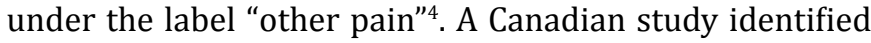
a prevalence of $66.0 \%$ of $\mathrm{OP}^{5}$. Severity of PD showed no association with OP level ${ }^{5}$. Mao et al. investigated possible associations between PD-related OP and other NMS ${ }^{6}$. They reported a prevalence of OP among PD cases of $47.9 \%$, and observed that PD patients with pain had more severe PD and scored higher in a depression questionnaire and lower in cognitive tests, with statistical significance ${ }^{6}$. In 2014, clinical differences between 39 PD patients with mutations in the glucocerebrosidase gene (GBA) and 539 sporadic PD controls were compared ${ }^{7}$. Four patients with GBAassociated PD and 16 controls with sporadic PD presented with $\mathrm{OP}$ as an initial symptom ${ }^{7}$. A single study evaluated OP in PD, multiple system atrophy (MSA) and progressive supranuclear palsy (PSP), reporting a greater prevalence in PD and MSA in comparison to PSP $(89.0 \%, 81.0 \%$ and $25.0 \%$, respectively), as well as a lower intensity of OP in $\mathrm{PSP}^{8}$.

\section{Neurotransmitter studies}

Tong et $\mathrm{al}^{9}$ found an inverse association between plasmatic levels of serotonin and 5-hydroxyindoleacetic acid and OP among PD patients, as well as lower plasmatic levels of both substances in PD patients when compared to healthy controls, and proposed that serotonergic dysfunction could play a role in $\mathrm{PD}$, particularly in the genesis of NMS like pain ${ }^{9}$. This research group had published an article one year earlier, investigating a possible correlation between PD NMS and plasmatic levels of glutamate, aspartate, $\gamma$-aminobutiric acid and glycine but, despite having found associations between plasma amino acids and depression and sleep disturbances, there was no significant correlation to $\mathrm{OP}^{10}$.

\section{Neurophysiologic studies}

One study using a quantitative technique to measure fiber-selective sensory thresholds compared the current perception and pain tolerance thresholds (CPT and PTT, respectively) between PD patients and controls ${ }^{11}$. CPT represents the intensity of an electrical stimulus at which the subject becomes aware of its presence, while PTT represents the intensity of such stimulus above which the subject perceives such stimulus as intolerable pain ${ }^{11}$. The method applied by the authors allows discrimination between $\mathrm{A} \beta$-, $\mathrm{A} \delta$ - and $\mathrm{C}$-fiber $\mathrm{CPT}$ and $\mathrm{PTT}^{11}$. It showed, with statistical significance, that patients had higher CPT for all three types of fibers and lower PTT for $A \beta$ and $A \delta$ fibers than controls. Patients who reported painful symptoms had statistically significant higher $\mathrm{A} \delta$ - and C-fiber CPT than those without pain. PD subjects with OP also had lower PTT, though without statistical significance ${ }^{11}$. These findings have led the authors to propose a role for abnormal sensory inputs in the pathogenesis of pain in $\mathrm{PD}^{11}$.

Another study applied a well-established conditioned pain modulation (CPM) paradigm to 25 PD patients and 30 controls $^{12}$. The paradigm combined painful heat stimuli and a cold pressor task as the conditioning stimulus ${ }^{12}$. No significant differences between both groups in pain modulation were found, and no significant effect of levodopa 
on CPM was shown ${ }^{12}$. Once CPM evaluates the efficacy of descending pain inhibition mechanisms, the authors concluded that PD is not associated with impairment of endogen pain inhibition beyond the age-related decline ${ }^{12}$.

\section{Studies with animal models}

Maegawa et al. analyzed the effects of nigrostriatal pathway lesions on nociception using a PD murine model ${ }^{13}$. Face rubbing in rats with chemical lesion of the left medial forebrain bundle was compared to that of control rats after nociceptive stimulus to the upper lip. Between 10 and 90 minutes after the stimulus, face rubbing was larger in PD-model rats ${ }^{13}$. From these data, it was inferred that nigrostriatal damage resulted in hypersensitivity to chemical painful stimulation. The authors suggested that dopamine may play a role in the modulation of inflammatory pain and central sensitization ${ }^{13}$.

\section{Pain lateralization in Parkinson's disease}

Our work reported a significant positive association between side of PD motor symptom onset and side of headache in those patients who reported unilateral headache ${ }^{2}$. Similarly, Rana et al. found an association between right-sided pain and PD motor symptoms onset on the right side ${ }^{5}$. Interestingly, such a relationship was not observed with left-sided pain ${ }^{5}$. Kresojević detected a high concordance between side of motor symptom onset and side of OP was high among the subjects who reported pain, with all 4 GBA cases and 14 out of 16 controls having OP localized to the upper limb ipsilateral to the motor symptoms onset, but the study only assessed pain as an initial symptom, and the number of PD patients who reported pain as the presenting symptom was small (20), and inferential analysis on this issue was not performed ${ }^{7}$. The study with PD-model rats has also addressed this issue, revealing that $\mathrm{PD}$-model rats showed hypersensitivity to painful stimuli, particularly worse ipsilaterally to the nigrostriatal involvement ${ }^{13}$.

In contrast, Mao found no significant relationship between side of motor symptom onset and side of $\mathrm{OP}^{6}$. Also, in the work of Chen, no association between sensory function or pain tolerance and the side of more severe motor symptoms was detected ${ }^{11}$.

Though the data available are limited and the methods employed in different studies vary, some studies have observed that pain syndromes occur more frequently in the same side as the motor symptoms in PD.

\section{Dopaminergic therapy and pain}

Rana did not find any significant associations between presence of dopaminergic therapies (DT) and pain scores, though $40 \%$ reported subjective improvement with the medication ${ }^{5}$. Simillarly, in Mao's work the daily equivalent dose of levodopa of the patients with OP was not higher than that of those without $\mathrm{OP}^{6}$. One study showed rates of subjective improvement of OP after DT of 51.0\%, 57.0\% and $25.0 \%$ for PD, MSA and PSP, respectively ${ }^{8}$. CPT and PTT were not affected by $\mathrm{DT}^{11}$, and DT did not influence $\mathrm{CPM}^{12}$. A proper comparison of these studies in regard to a possible effect of DT on pain in PD is difficult, because the methods and study designs are heterogeneous. Nonetheless, most of them suggest DT has no objective effect on pain. A randomized controlled trial of DT in PD having as primary outcome the reduction of OP would perhaps clarify this issue.

The effects of deep brain stimulation on pain in Parkinson's disease

Deep brain stimulation (DBS) has emerged as an effective treatment for motor symptoms of $\mathrm{PD}^{14}$. Its impact on NMS of PD has been increasingly investigated, providing new insights into the pathophysiology of pain in PD.

A cohort followed 41 PD patients who underwent subthalamic nucleus DBS (STN-DBS), assessing OP and NMS before and one year after surgery ${ }^{14}$. An improvement of OP after STN-DBS was shown, particularly of dystonic and musculoskeletal pain ${ }^{14}$. Neuropathic and central pain were not influenced by the procedure ${ }^{14}$. The authors did not find any association between motor symptom improvement and $\mathrm{OP}$ reduction after $\mathrm{STN}-\mathrm{DBS}^{14}$. A previous study with a similar design had found a significant reduction of sensory and affective aspects of pain in a sample of 58 PD patients after STN-DBS, and that effect was independent of motor improvement ${ }^{15}$. Another work followed and evaluated OP in PD patients for 8 years after STN-DBS, documenting an improvement of OP in all 24 subjects, though no objective method for pain intensity quantification was employed ${ }^{16}$. It also reported that 3 out of 24 selected PD patients had headache ${ }^{16}$. A cohort published in 2015 reported an improvement of OP and lower back pain in PD patients after STN-DBS ${ }^{17}$.

It has been consistently shown that STN-DBS may reduce $\mathrm{OP}^{14-17}$, and that effect seems to be independent of the procedure's benefit on parkinsonian motor symptoms ${ }^{14,15}$. Once DBS mainly acts on the nigrostriatal pathways via STN stimulation, thus increasing thalamocortical excitation, it seems plausible that basal ganglia may play a direct role in pain modulation.

\section{Conclusion}

Even with a high prevalence of OP among PD patients ${ }^{4-6}$, headache itself does not seem to be a common complain ${ }^{4,16}$. That is in accordance with our case-control study, in which a lower occurrence of headache was observed in PD subjects $^{2}$. Both headache and OP may be more frequently ipsilateral to motor symptoms in $\mathrm{PD}^{2,5,7,13}$, though study 
results are conflicting and data are lacking to make a definite assumption.

The genesis of pain in PD may be more related to abnormal sensory input pathways ${ }^{11}$ than to impaired pain-inhibiting mechanisms ${ }^{12}$. In the animal model of $\mathrm{PD}$, nigrostriatal damage was shown to produce central sensitization ${ }^{13}$. Serotonergic dysfunction may also be involved in the pathophysiology of pain in $\mathrm{PD}^{9}$.

Although the analysis of DT on pain in PD did not show any significant association ${ }^{5,6,8,11}$, STN-DBS was shown to reduce pain in $\mathrm{PD}^{14-17}$, and that reduction seems to be independent of the benefit of DBS on motor symptoms ${ }^{14,15}$. Once STN-DBS increases thalamocortical activation, these findings could mean that the basal ganglia may participate in pain modulation mechanisms that, when dysfunctional, could lead to the generation of pain in PD independently of motor symptoms.

The reason why headache is so uncommon in PD despite the high frequency of $O P$ is still elusive. PD impairment of pain modulation circuits may be selective for certain painful syndromes, increasing the occurrence of certain kinds of pain while decreasing others. Data about headache in PD is scarce and more comprehensive and high-quality descriptive studies are necessary in order to better characterize this symptom and its occurrence in PD. Conversely, studies employing functional imaging to compare PD patients and controls in regard to patterns of brain activation to painful stimuli could a promote a better comprehension of the mechanisms through which PD affects pain modulation.

\section{References}

1. Ford B. Pain in Parkinson's disease. Mov Disord 2010; 25 Suppl 1: S98-103.

2. Nunes JC, Costa Bergamaschi EN, Freitas FC, Diaz AP, Queiroz LP, Debona R, et al. Prevalence of headache in patients with Parkinson's disease and its association with the side of motor symptom onset. Neurol Sci 2014 Apr; 35(4): 595-600.

3. Queiroz LP, Peres MF, Piovesan EJ, Kowacs F, Ciciarelli MC, Souza JA, et al. A nationwide population-based study of migraine in Brazil. Cephalalgia 2009; 29(6): 642-9.

4. Valkovic P, Minar M, Singliarova H, Harsany J, Hanakova M, Martinkova J, Benetin J. Pain in Parkinson's Disease: A Cross-Sectional Study of Its
Prevalence, Types, and Relationship to Depression and Quality of Life. PLoS One 2015; 10(8): e0136541.

5. Rana A, Saeed U, Masroor MS, Yousuf MS, Siddiqui I. A cross-sectional study investigating clinical predictors and physical experiences of pain in Parkinson's disease. Funct Neurol 2013; 28(4): 297-304.

6. Mao CJ, Chen JP, Zhang XY, Chen Y, Li SJ, Li J, et al. Parkinson's disease patients with pain suffer from more severe NMS. Neurol Sci 2015; 36(2): 263-8.

7. Kresojević N, Janković M, Petrović I, Kumar KR, Dragašević N, Dobričić $\mathrm{V}$, et al. Presenting symptoms of GBA-related Parkinson's disease. Parkinsonism Relat Disord 2015 Jul; 21(7): 804-7.

8. Kass-Iliyya L, Kobylecki C, McDonald KR, Gerhard A, Silverdale MA. Pain in multiple system atrophy and progressive supranuclear palsy compared to Parkinson's disease. Brain Behav 2015 May; 5(5): e00320.

9. Tong Q Zhang L, Yuan Y, Jiang S, Zhang R, Xu Q, et al. Reduced plasma serotonin and 5-hydroxyindoleacetic acid levels in Parkinson's disease are associated with nonmotor symptoms. Parkinsonism Relat Disord 2015 Aug; 21(8): 882-7.

10. Tong Q, Xu Q, Xia Q, Yuan Y, Zhang L, Sun H, et al. Correlations between plasma levels of amino acids and nonmotor symptoms in Parkinson's disease. J Neural Transm (Vienna) 2015 Mar; 122(3): 411-7.

11. Chen Y, Mao CJ, Li SJ, Wang F, Chen J, Zhang HJ, et al. Quantitative and fiber-selective evaluation of pain and sensory dysfunction in patients with Parkinson's disease. Parkinsonism Relat Disord 2015 Apr; 21(4): 361-5.

12. Grashorn W, Schunke O, Buhmann C, Forkmann K, Diedrich S, Wesemann K, Bingel U. Influence of Dopaminergic Medication on Conditioned Pain Modulation in Parkinson's Disease Patients. PLoS One 2015 Aug 13; 10(8): e0135287.

13. Maegawa H, Morimoto Y, Kudo C, Hanamoto H, Boku A, Sugimura M, et al. Neural mechanism underlying hyperalgesic response to orofacial pain in Parkinson's disease model rats. Neurosci Res 2015 Jul; 96: 59-68.

14. Cury RG, Galhardoni R, Fonoff ET, Dos Santos Ghilardi MG, Fonoff F, Arnaut D, et al. Effects of deep brain stimulation on pain and other nonmotor symptoms in Parkinson disease. Neurology. 2014 Oct 14; 83(16): 1403-9.

15. Pellaprat J, Ory-Magne F, Canivet C, Simonetta-Moreau M, Lotterie JA, Radji F, et al. Deep brain stimulation of the subthalamic nucleus improves pain in Parkinson's disease. Parkinsonism Relat Disord 2014 Jun; 20(6): 662-4.

16. Jung YJ, Kim HJ, Jeon BS, Park H, Lee WW, Paek SH. An 8-Year Followup on the Effect of Subthalamic Nucleus Deep Brain Stimulation on Pain in Parkinson Disease. JAMA Neurol 2015 May; 72(5): 504-10.

17. Smith H, Gee L, Kumar V, Ramirez-Zamora A, Durphy J, Hanspal E, et al. Deep brain stimulation significantly decreases disability from low back pain in patients with advanced Parkinson's disease. Stereotact Funct Neurosurg 2015; 93(3): 206-11. 